\title{
Matching microlensing events with X-ray sources
}

\author{
N. Sartore ${ }^{1,2}$ and A. Treves ${ }^{2,3}$ \\ 1 INAF - Istituto di Fisica Spaziale e Fisica Cosmica, via Bassini 15, 20133 Milan, Italy \\ e-mail: sartore@iasf-milano.inaf.it \\ 2 Dipartimento di Fisica e Matematica, Università dell'Insubria, via Valleggio 11, 22100 Como, Italy \\ 3 Affiliated to INAF and INFN
}

Received 10 May 2011 / Accepted 18 December 2011

\begin{abstract}
Aims. The detection of old neutron stars and stellar mass black holes in isolation is one of the most sought after goals of compact object astrophysics. Microlensing surveys may help in achieving this aim because the lensing mechanism is independent of the emission properties of the lens. Several black hole candidates have indeed been detected by means of microlensing observations have been reported in the literature. The identification of counterparts, especially in the X-rays, would be a strong argument in favor of the compact nature of these lenses.

Methods. We perform a cross-correlation between the catalogs of microlensing events produced by the OGLE, MACHO, and MOA teams, and those of X-rays sources from the data acquired by the XMM-Newton and Chandra satellites. On the basis of our previous work, we select only microlensing events with durations longer than one hundred days, which should contain a large fraction of lenses as compact objects. Our matching criterion takes into account the positional coincidence on the sky.

Results. We find a single match between a microlensing event, OGLE-2004-BLG-081 ( $t_{\mathrm{E}} \sim 103$ days), and the X-ray source 2XMM J180540.5-273427. The angular separation is $\sim 0.5$ arcsec, i.e. well within the $90 \%$ error box of the X-ray source. The hardness ratios reported in the $2 \mathrm{XMM}$ catalog imply that it has a hard spectrum with a peak between $2 \mathrm{keV}$ and $4.5 \mathrm{keV}$ or it has a softer but highly absorbed spectrum. Moreover, the microlensing event is not fully constrained, and other authors propose a possible association of the source star with either a flaring cataclysmic variable or a RS Canum Venaticorum-like star.

Conclusions. The very small angular separation (within uncertainties) is a strong indicator that 2XMM J180540.5-273427 is the X-ray counterpart of the OGLE event. However, the uncertainties in the nature of both the lensed system and the lens itself challenge the interpretation of 2XMM J180540.5-273427 as the first confirmed isolated black hole identified so far. In any case, it verifies the diagnostic capacity of microlensing surveys and open the pathway for further identifications of black hole or neutron star candidates.
\end{abstract}

Key words. stars: neutron - surveys - catalogs - virtual observatory tools - gravitational lensing: micro

\section{Introduction}

The Galactic population of neutron stars and and black holes (NSs and BHs hereafter) is expected to consist of as many as $10^{8}-10^{9}$ objects (e.g. Keane \& Kramer 2008). This population is almost undetected with the exception of a few thousands of sources, which are mainly isolated young NSs emitting as radio/X-ray/gamma-ray pulsars and NSs and BHs in X-ray binaries.

Observing the vast majority of old, likely isolated objects is difficult because the proposed recycling mechanism, i.e. residual accretion from the interstellar medium (e.g. Ostriker et al. 1970; Treves \& Colpi 1991), is impeded by many factors, such as the large velocities (e.g. Treves et al. 2000; Popov et al. 2000; Sartore et al. 2010), the presence of the magnetic field in NSs (e.g. Toropina et al. 2003; Perna et al. 2003) and, for BHs, the small radiation efficiencies of the accretion process (e.g. Agol \& Kamionkowski 2002; Beskin \& Karpov 2005). Nevertheless, old isolated remnants (NSs and BHs) can certainly act as gravitational lenses.

Gravitational microlensing (ML) events occur when light from a star is deflected and amplified by a foreground mass (Paczynski 1986). Several thousands of ML events have indeed been observed so far (see Moniez 2010, for a review). Since most of the events are due to stars with masses lower than one solar mass, NSs and BHs contribute essentially to the long duration events, the longest ones being associated with BHs (e.g. Mao et al. 2002; Bennett et al. 2002). According to Bennett et al. (see also Calchi Novati et al. 2008), the number of long duration events is in excess of that inferred from theoretical models (e.g. Gould 2000; Wood \& Mao 2005). Sartore \& Treves (2010) demonstrated that this excess can be explained assuming that $\mathrm{BHs}$ receive large natal kicks at birth, of the same magnitude as those observed in NSs (e.g. Hobbs et al. 2005; Gualandris et al. 2005). Under this assumption, about 40 percent of the events longer than 100 days are due to BHs.

Thus, microlensing surveys may have already detected tens of events related to NSs and BHs. However, the nature of candidate $\mathrm{BH}$ lenses has to be assessed with independent methods. The mass estimated from ML events is in general affected by large uncertainties, given the degeneracy of the timescale with the input parameters such as the mass of the lens and the geometry of the source-lens-observer system, i.e. the relative positions and velocities. However, the detection of X-rays coming from the position of the ML event would be a strong indication of a compact object. A search for the X-ray counterpart of one of the most promising $\mathrm{BH}$ candidates detected by means of ML was performed by Nucita et al. (2006, see also Agol \& Kamionkowski 2002). However, a 100 ks pointing with $X M M$-Newton gave no result down to a limiting flux of $\sim 10^{-15} \mathrm{erg} \mathrm{s}^{-1}$ in the $0.2-10 \mathrm{keV}$ energy range. 
In this study, we present a systematic cross-correlation analysis of long-duration ML events with the recent catalogs of X-ray sources produced with the data collected by the XMM-Newton and Chandra satellites. The paper is organized as follows. Section 2 briefly describes the expected spectra of NSs and BHs accreting at low rates. In Sect. 3, we report on the list of ML events and catalogs of X-ray sources used to find possible counterparts. A description of the cross-correlation method is also given. In Sect. 4, we present our results and briefly discuss a source matching the cross-correlation criterion. Finally in Sect. 5 we summarize the main points of the paper and discuss the possibilities of finding more remnant candidates with ML.

\section{Expected X-ray spectra of accreting remnants}

It is commonly accepted that isolated NSs and BHs accreting from the ISM should radiate mostly in the X-ray band. For instance, the spectrum emitted by a NS accreting at a low rate could be roughly approximated by a Planckian, with a temperature of few hundreds electronvolts (Ostriker et al. 1970; Treves \& Colpi 1991; Blaes \& Madau 1993). More detailed calculations (Zampieri et al. 1995; Zane et al. 2000) showed that the spectrum emerging from an accreting NS, either magnetized or non-magnetized, could differ substantially from a blackbody and in particular the resulting spectrum be harder than the blackbody emission at the same accretion rate. However, the overall behavior still resembles a Planckian-like spectrum with peak emission in the soft X-rays.

The situation for BHs is quite different since, at variance with NSs, the efficiency of the conversion of accreted matter into radiation is expected to be lower. Models of ISM accretion onto isolated BHs were developed by Agol \& Kamionkowski (2002), Beskin \& Karpov (2005), and Mapelli et al. (2006). The underlying physics is in general assumed to be similar to that of $\mathrm{BH}$ candidates in X-ray binaries but the exact details remain poorly constrained. Nevertheless, the emerging spectra should have both thermal-like and non thermal components, as observed in X-ray binaries.

The X-ray luminosities of accreting NSs/BHs depend strongly on the relative velocity of the compact object NS/BH with respect to the surrounding medium (e.g. Bondi 1952)

$L_{X} \sim 10^{31} \eta\left(\frac{n}{1 \mathrm{~cm}^{-3}}\right)\left(\frac{v}{10 \mathrm{~km} \mathrm{~s}^{-1}}\right)^{-3}\left(\frac{M}{1 M_{\odot}}\right)^{3} \mathrm{erg} \mathrm{s}^{-1}$,

where $\eta$ is the efficiency of the X-ray emission, $n$ is the number density of the ISM, and $v$ and $M$ are the relative velocity with respect to the surrounding medium and the mass of the compact object, respectively. Old, but rapidly moving NSs are expected to populate the Galactic disk with velocities of $\sim 200 \mathrm{~km} \mathrm{~s}^{-1}$ (e.g. Sartore et al. 2010). The resulting luminosity is of approximately $10^{27}-10^{28} \mathrm{erg} \mathrm{s}^{-1}$. For BHs, the effect of their higher mass is counterbalanced by the lower energy conversion efficiency, thus the nominal X-ray luminosities are approximately of the same order as accreting NSs (see however the paper of Mapelli et al.). In addition, when comparing with observations one has to take into account the absorption effect, which can be significant for soft X-rays.

\section{Method}

\subsection{Selection of microlensing events}

We based our present study on the available public data of the OGLE (Udalski et al. 1992), MACHO (Alcock et al. 1993) and
MOA (Muraki et al. 1999) collaborations. Following the results of Sartore \& Treves, we selected only events with timescales longer than 100 days. The OGLE (Optical Gravitational Lensing Experiment) data ${ }^{1}$ were collected with the Early Warning System (EWS, see Udalski 2003) from 1998 to 2009 and correspond to the OGLE-II and OGLE-III phases of the survey. The number of events detected in the first year (1998) was 41, of which none had a duration longer than 100 days, while for instance in 2008 the number of events detected was 654 with 38 events longer than 100 days. The total number of events from OGLE is 4117, of which 177 percent fall in our range of interest. The MACHO (MAssive Compact Halo Objects) survey data $^{2}$ were collected from 1993 to 1999 and are comprehensive for 528 bulge events reported by Thomas et al. (2005), as well as the red clump giants events reported by Popowski et al. (2005), totaling 567 events. Of these, 69 events are longer than 100 days. The MOA (Microlensing Observations in Astrophysics) survey ${ }^{3}$ started in 2000, and in 2006 entered its second phase. While the number of events detected in 2000 was barely 8 , the current detection rate is similar to that of the OGLE-III survey, i.e. $\sim 500-600 \mathrm{yr}^{-1}$. The number of events detected by MOA up to 2010 is 2622 , of which 268 have timescales longer than 100 days.

\subsection{X-ray sources catalogs}

We searched for X-ray counterparts of our selected sample of ML events in the version 1.2 of the second XMM-Newton Serendipitous Source Catalog (2XMM hereafter, Watson et al. 2009) and in version 1.1 of the Chandra Source Catalog (CSC hereafter, Evans et al. 2010). The most recent version of the XMM catalog ${ }^{4}$ was released in April 2010. It contains data of 191870 unique sources with a median flux (in the $0.2-12 \mathrm{keV}$ band) $\sim 2.5 \times 10^{-14} \mathrm{erg} \mathrm{s}^{-1} \mathrm{~cm}^{-2}$, with 20 percent of the sources having fluxes below $10^{-14} \mathrm{erg} \mathrm{s}^{-1} \mathrm{~cm}^{-2}$. The CSC catalog ${ }^{5}$, released in August 2010, covers approximately 320 square degrees of the sky at the $10^{-13} \mathrm{erg} \mathrm{s}^{-1} \mathrm{~cm}^{-2}$ flux limit $(0.5-7.0 \mathrm{keV}$ band). The sky coverage drops to $\sim 6$ square degrees for a flux limit of $10^{-15} \mathrm{erg} \mathrm{s}^{-1} \mathrm{~cm}^{-2}$ (Evans et al. 2010). The total number of unique sources contained in the catalog is 106586 .

The OGLE-III survey monitored a region of the sky comprised between $l=-2^{\circ}$ and $l=-7^{\circ}$ for $b= \pm 10^{\circ}$, i.e. $\sim 100$ square degrees. The area covered by the MACHO and MOA-II surveys is roughly half of that of OGLE-III (e.g. Thomas et al. 2005). The XMM-Newton and Chandra surveys performed respectively 17 and 5 pointings towards the same $\operatorname{area}^{6}$ (see Fig. 1), which, considering the $30^{\prime} \times 30^{\prime}$ field of view of the EPIC cameras on board XMM-Newton and the $16^{\prime} \times 16^{\prime}$ field of view of the ACIS cameras on board Chandra, correspond to $\sim 14$ and $\sim 1$ square degrees, respectively.

\subsection{Cross-correlation analysis}

Given the large uncertainties affecting the expected observational appearance of old isolated NSs and BHs, we did not apply

\footnotetext{
${ }^{1}$ http://ogle.astrouw.edu.pl/

2 http://wwwmacho.mcmaster.ca/

${ }^{3}$ http://www.phys. canterbury.ac.nz/moa/

${ }^{4}$ http://xmmssc-www.star.le.ac.uk/Catalogue/

xcat_public_2XMM.html

5 http://cxc.harvard.edu/csc/

${ }^{6}$ Multiple observations of the same region are considered as a single pointing.
} 

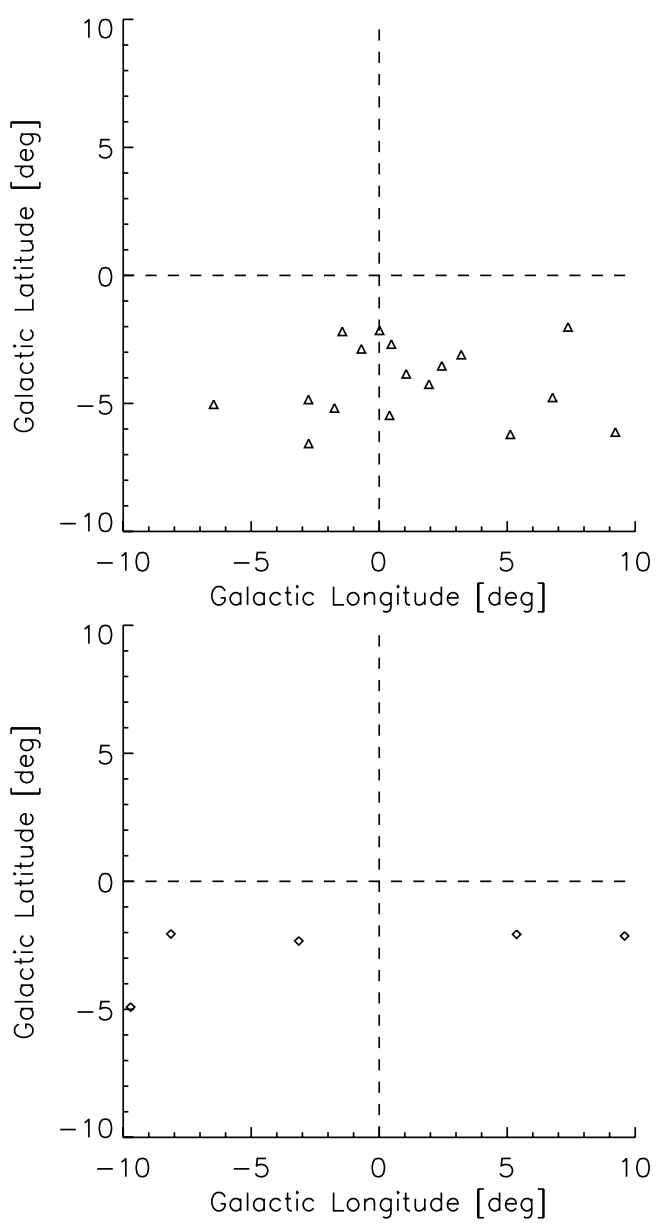

Fig. 1. Centroids of the pointings, in Galactic coordinates $(l, b)$, performed by the XMM-Newton (top) and Chandra (bottom) satellites in the region of the sky monitored by the OGLE-III survey (see text).

any particular spectral or variability criteria to the possible X-ray counterparts. Nevertheless, we removed spurious sources in the 2XMM catalog by selecting only those with $S U M \_F L A G=0$, i.e. those for which none of the warning flags of the EPIC instruments were set. These flags indicate the probability of a detected source being spurious. For the CSC catalog there is no analogue flag, but the estimated number of spurious sources is expected to be less than one in every field with $100 \mathrm{ks}$ of integration. Thus, our matching criterion was based only on the positional coincidence of the long timescale ML events with the X-ray sources of the 2XMM and CSC catalogs.

Our cross-correlation software computes the projected distance between the selected ML events and the entries of both the 2XMM and CSC catalogs. A positive match is found when a ML event lies with the $3 \sigma$ error circle of an X-ray source. Positional errors of single sources are taken from the respective catalogs. The positional error of 2XMM sources (POS ERR column) already accounts for systematic errors. The total $1 \sigma$ uncertainty is calculated as the squared root of the sum of statistical and systematic errors (Watson et al. 2009)

$P O S E R R=\sqrt{R A D E C_{-} E R R^{2}+S Y S E R R^{2}}$.

To take into account the systematic error $(0.16$ arcsec $)$ of CSC sources, we used the equation suggested by the CSC team

err_ellip_r $r_{0, \mathrm{tot}}=2.4477467$

$$
\times \sqrt{0.1669041 \times\left(\text { err_ellip_r } r_{0, \mathrm{cat}}\right)^{2}+0.0256} \text {. }
$$

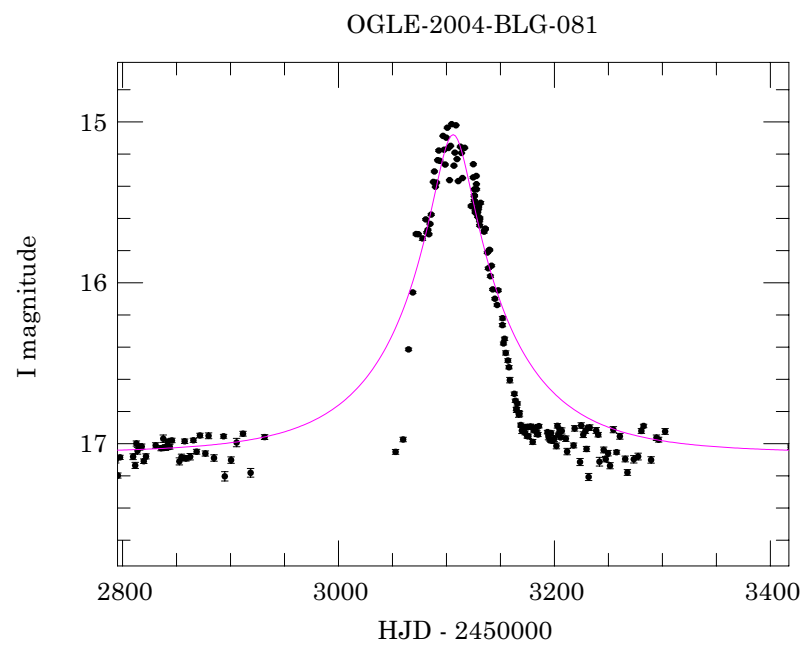

Fig. 2. Light curve of the event OGLE-2004-BLG-081. The solid line represents the best fit to the light curve assuming a standard microlensing model. Source: OGLE website.

The typical statistical error for on-axis CSC sources is $\sim 0.2$ arcsec, while for off-axis sources at 14 arcmin the statistical error is $\sim 3.5$ arcsec. We assumed that the positional error of the ML events was $\sigma_{\mathrm{ML}} \sim 1.5$ arcsec for all the events. Thus, the resulting radius of the error circle was assumed to be the root mean square of the X-ray source and ML event positional uncertainties

$\sigma=\sqrt{\sigma_{\mathrm{ML}}^{2}+\sigma_{\mathrm{X}}^{2}}$

\section{Results}

The cross-correlation analysis returned a single positive match in the 2 XMM catalog. The associated lensing event was observed in 2004 by both the OGLE and MOA surveys and was identified with the OGLE-2004-BLG-081 and MOA 2004-BLG-3 events, respectively. The duration of the event reported by the OGLE team is about 103.63 days. However, the light curve is poorly fitted by standard lensing models (Fig. 2). Wyrzykowski et al. (2006) found that the baseline of the source star, i.e. its magnitude when not lensed $(I \sim 17)$, has a suspected periodicity of $\sim 4$ days, implying that it is an eclipsing binary. In particular, the shape of the folded light curve is indicative of a contact binary system.

To add to the confusion, the MOA team reported a baseline $I \sim 8$ (sic!), a duration of $\sim 6.73$ days, and an amplification very close to unity, $A \sim 1.002$. However, a visual inspection of the stellar field does not confirm that there is such a bright star, whose image would have been affected by diffraction. Thus, we rely exclusively on the OGLE data to characterize the event.

The X-ray source associated with the ML event, 2XMM J180540.5-273427 (J1805 hereafter), was serendipitously observed during a pointing of MACHO-96-BLG-5, another $\mathrm{BH}$ candidate detected by means of microlensing (Bennett et al. 2002; Nucita et al. 2006). The X-ray properties of the source were retrieved with the XCat-DB web interface (Motch et al. 2009). The total number of counts is $312.744 \pm$ $0.001(0.2-12 \mathrm{keV}$ band) corresponding to a flux of (3.39 \pm $0.78) \times 10^{-14} \mathrm{erg} \mathrm{s}^{-1} \mathrm{~cm}^{-2}$, which implies a luminosity, neglecting the photoelectric absorption of the ISM, of $\sim 3 \times$ $10^{30}(d / 1 \mathrm{kpc})^{2} \mathrm{erg} \mathrm{s}^{-1}$. If $\mathrm{J} 1085$ were responsible for the magnification of a bulge star $(d \sim 8 \mathrm{kpc})$, then it should be at an 
A\&A 539, A52 (2012)

Table 1. Observed properties of the X-ray source 2XMM J180540.5-273427.

\begin{tabular}{lccccc}
\hline \hline $\begin{array}{l}\text { Energy band } \\
{[\mathrm{keV}]}\end{array}$ & $0.2-0.5$ & $0.5-1.0$ & $1.0-2.0$ & $2.0-4.5$ & $4.5-12$ \\
\hline $\begin{array}{l}\text { Flux } \\
{\left[\times 10^{-14} \mathrm{erg} \mathrm{s}^{-1} \mathrm{~cm}^{-2}\right]}\end{array}$ & $0.002 \pm 0.001$ & $0.034 \pm 0.027$ & $0.298 \pm 0.050$ & $1.20 \pm 0.146$ & $1.73 \pm 0.762$ \\
\hline Hardness ratio & $0.982 \pm 0.268$ & $0.738 \pm 0.141$ & $0.132 \pm 0.100$ & $-0.356 \pm 0.157$ & \\
\hline
\end{tabular}

Notes. The data are retrieved from the XCat database. Hardness ratios are calculated as described in Watson et al., with the lower energy band corresponding to the position in the table.

intermediate distance and its X-ray luminosity should be lower than $\sim 10^{32} \mathrm{erg} \mathrm{s}^{-1}$.

The positional uncertainty of the source is $\sim 2 \operatorname{arcsec}$ and it lies about 0.5 arcsec from the position of the ML event. We report the fluxes for the different EPIC bands and the relative hardness ratios in the XCat database in Table 1.

\section{Discussion and conclusions}

The small angular separation $(\sim 0.5 \operatorname{arcsec})$ between the position of the ML event OGLE-2004-BLG-081 and J1805 is smaller than the $1 \sigma$ positional uncertainty of the X-ray source and makes the association highly likely. Thus, if J1805 were actually a BH, this would prove that ML surveys can detect isolated compact objects. However, there are a number of uncertainties that need to be addressed in order to accept the claim with some degree of confidence.

First, the nature of the event reported by the OGLE and MOA surveys is unclear. As already pointed out, the shape of the light curve would rule out a ML event, even accounting for secondary effects such as parallax or blending. That the source star is possibly a contact binary implies that it is a cataclysmic variable $(\mathrm{CV})$, i.e. contains an accreting white dwarf. This would imply that the event was in reality an outburst episode rather than a genuine gravitational lens. An accreting white dwarf would easily explain the detected X-ray radiation as emission from the matter accreted by the degenerate star. However, the duration of the putative outburst and its lightcurve are unusual for this kind of sources (see e.g. Butler 1991; Kuulkers et al. 2006), thus challenging the CV hypothesis.

Alternatively, it has been suggested that the lensed star is a chromospherically active variable (Bernhard 2009), possibly of the RS Canum Venaticorum (RS CVn) type. This class of variables is known to show periodic variations that are thought to be related to the active regions on the surface of the star or the ellipticity of the star itself. These effects can mimic the lightcurve of an eclipsing binary and explain the observed modulation of the baseline. Furthermore, RS CVn stars are known X-ray emitters, with luminosities of $\sim 10^{31} \mathrm{erg} \mathrm{s}^{-1}$, and also show flaring activity at both optical and X-ray wavelengths. Nevertheless, also in this case the amplitude, shape, and duration of the optical event are unusual for RS CVn stars (Shore, priv. comm.).

In addition to the uncertain classification of the optical event, the nature of the X-ray source J1805 is also poorly constrained. The small number of collected photons does not allow a reliable characterization of the spectrum. The hardness ratios are positive at low energies and change sign between $2 \mathrm{keV}$ and $4.5 \mathrm{keV}$, which is indicative of either a hard or highly absorbed spectrum. A hard spectrum would rule out a NS as the accreting object since its temperature is expected to be below $\sim 1 \mathrm{keV}$. Thus, if J1805 were an isolated compact object, it should be a BH.
In conclusion, we have performed a cross-correlation analysis of long timescale ML events with the soft X-ray sources of the recently released XMM-Newton and Chandra catalogs. We report a positive match between the ML event OGLE-2004BLG-081 and the X-ray source 2XMM J180540.5-273427. The nature of both the ML event and the the X-ray source are still unclear. Follow-up optical and X-ray observations of the field of 2XMM J180540.5-273427 at high angular resolution could help us to characterize the source star as well as the X-ray source.

We stress that, even if the association between OGLE-2004BLG-081 and 2XMM J180540.5-273427 would not be confirmed, the argument that a large fraction of long duration events are related to BHs is still valid. As shown in Sect. 2, and in particular in Eq. (1), the expected flux from an isolated BH or NS depends on many unknown quantities such as the velocity of the collapsed object, the density of the ISM, the photoelectric absorption etc. Therefore, it is not straightforward to use the absence, or paucity, of a correlation between the X-ray sources and the ML events as a constraint on so many parameters.

In the next few years, we expect a substantial enlargement of the catalogs of ML events and X-ray sources. In particular the eROSITA mission, which should be launched shortly, will make a survey of the entire sky that in the soft X-ray band $(0.5-2 \mathrm{keV})$ will be 30 times more sensitive than ROSAT. At the same time, deep systematic exposures with Chandra and XMM-Newton, of the most promising ML events, chosen mainly on the basis of their duration, will also help us to place stronger constraints on the flux of accreting isolated compact objects.

Note added in proofs: The OGLE team (Wyrzykowski \& Poleski, priv. comm.) reported that OGLE-2004-BLG-081 is very likely not a microlensing event and that the OGLE data strongly favors a dwarf nova scenario.

Acknowledgements. The work presented in this paper was made possible thanks to the public data from the OGLE, MACHO, and MOA microlensing surveys and from the X-ray source catalogs of the XMM-Newton and Chandra satellites. We thank the anonymous referee for helpful comments and suggestions, which improved the previous version of this paper. NS also thanks A. Paizis for useful discussion. The work of NS is supported by ASI-INAF through grant I/009/10/0.

\section{References}

Agol, E., \& Kamionkowski, M. 2002, MNRAS, 334, 553

Agol, E., Kamionkowski, M., Koopmans, L. V. E., \& Blandford, R. D. 2002, ApJ, 576, L131

Alcock, C., Akerlof, C. W., Allsman, R. A., et al. 1993, Nature, 365, 621

Bennett, D. P., Becker, A. C., Quinn, J. L., et al. 2002, ApJ, 579, 639

Bernhard, K. 2009, Open European Journal on Variable Stars, 117, 1

Beskin, G. M., \& Karpov, S. V. 2005, A\&A, 440, 223

Blaes, O., \& Madau, P. 1993, ApJ, 403, 690

Bondi, H. 1952, MNRAS, 112, 195

Butler, C. J. 1991, Mem. Soc. Astron. It., 62, 243

Calchi Novati, S., de Luca, F., Jetzer, P., Mancini, L., \& Scarpetta, G. 2008, A\&A, 480, 723

Evans, I. N., Primini, F. A., Glotfelty, K. J., et al. 2010, ApJS, 189, 37 
N. Sartore and A. Treves: Matching microlensing events with X-ray sources

Gould, A. 2000, ApJ, 535, 928

Gualandris, A., Colpi, M., Portegies Zwart, S., \& Possenti, A. 2005, ApJ, 618, 845

Hobbs, G., Lorimer, D. R., Lyne, A. G., \& Kramer, M. 2005, MNRAS, 360, 974

Keane, E. F., \& Kramer, M. 2008, MNRAS, 391, 2009

Kuulkers, E., Norton, A., Schwope, A., \& Warner, B. 2006, in Compact Stellar X-Ray Sources, ed. W. H. G. Lewin \& M. van der Klis (Cambridge University Press) [arXiv: astro-ph/0302351]

Mao, S., Smith, M. C., Woźniak, P., et al. 2002, MNRAS, 329, 349

Mapelli, M., Ferrara, A., \& Rea, N. 2006, MNRAS, 368, 1340

Moniez, M. 2010, Gen. Rel. Grav., 42, 2047

Motch, C., Michel, L., \& Pineau, F.-X. 2009, Astronomical Data Analysis Software and Systems XVIII, 411, 466

Muraki, Y., Sumi, T., Abe, F., et al. 1999, Progr. Theoret. Phys. Suppl., 133, 233

Nucita, A. A., De Paolis, F., Ingrosso, G., et al. 2006, ApJ, 651, 1092

Ostriker, J. P., Rees, M. J., \& Silk, J. 1970, Astrophys. Lett., 6, 179

Paczynski, B. 1986, ApJ, 304,

Perna, R., Narayan, R., Rybicki, G., Stella, L., \& Treves, A. 2003, ApJ, 594, 936
Popov, S. B., Colpi, M., Treves, A., et al. 2000, ApJ, 530, 8

Popowski, P., Griest, K., Thomas, C. L., et al. 2005, ApJ, 631, 879

Sartore, N., \& Treves, A. 2010, A\&A, 523, A33

Sartore, N., Ripamonti, E., Treves, A., \& Turolla, R. 2010, A\&A, 510, A23

Thomas, C. L., Griest, K., Popowski, P., et al. 2005, ApJ, 631, 906

Toropina, O. D., Romanova, M. M., Toropin, Y. M., \& Lovelace, R. V. E. 2003, ApJ, 593, 472

Treves, A., \& Colpi, M. 1991, A\&A, 241, 107

Treves, A., Turolla, R., Zane, S., \& Colpi, M. 2000, PASP, 112, 297

Udalski, A., Szymanski, M., Kaluzny, J., Kubiak, M., \& Mateo, M. 1992, Acta Astron., 42, 25

Udalski, A. 2003, Acta Astron., 53, 291

Watson, M. G., Schröder, A. C., Fyfe, D., et al. 2009, A\&A, 493, 339

Wood, A., \& Mao, S. 2005, MNRAS, 362, 945

Wyrzykowski, L., Udalski, A., Mao, S., et al. 2006, Acta Astron., 56, 145

Zampieri, L., Turolla, R., Zane, S., \& Treves, A. 1995, ApJ, 439, 849

Zane, S., Turolla, R., \& Treves, A. 2000, ApJ, 537, 387 